\title{
Characteristics and Interventions of Bullying Behavior of Teenagers under the Background of Informatization
}

\author{
Junping Liu \\ Faculty of general education, Beijing Information Technology College, Beijing, China
}

\begin{abstract}
With the rapid development of industrialization and informatization, the significant improvement of human civilization, people pay much more attention to education and mental health, especially the mental health of teenagers. In recent years, the impact of campus bullying on adolescents' physical and mental health has aroused widespread social concern. A self-designed questionnaire is used to investigate 392 teenagers in the research. The characteristics and related factors of bullying and being bullied of student among three grades are explored, and targeted intervention measures are explored. The result shows that compared with senior students, junior students are more involved in bullying behavior. Bullying behavior is closely related to class factors: bullying behavior often starts from classmates, and occurs mostly in classrooms. Interpersonal relationship, affection to the class is negatively correlated with the frequency of bullying and being bullied. A multi-level intervention system of "school, class and individual" need to develop mainly by prophase prevention, class construction and peer counseling.
\end{abstract}

Keywords: Teenagers, Bullying behavior, Characteristics, Intervention measures, informatization

\section{Introduction}

In the era of rapid development of informatization, the characteristics of teenagers' interpersonal communication have changed. With so many social APP, their interpersonal communication is faster and more convenient, but also more indirect and superficial. The use of the Internet weakens the sense of responsibility, moral sense and trust of interpersonal interaction, and alienates the interaction in the real society, making it difficult for them to form a stable and in-depth interpersonal interaction[1]. Under such a background, teenagers are more insecure and trustless in interpersonal communication, and are more likely to be involved in bullying.

Bullying is a special type of aggressive behavior; it refers to the aggressive behavior repeatedly carried out by the stronger ones against the weaker ones [2]. Studies have shown that bullying is universal and harmful among teenagers [3]. When bullying happens, not only the students involved in bullying are hurt physically and mentally, but also the students on the sidelines are impacted. In recent years, campus bullying incidents have been reported frequently, and have aroused widespread social concern. It is of great significance for teenagers' health and campus culture construction to analyze the characteristics of bullying and intervene in a targeted way.

\section{Research Method}

In the first stage, questionnaire and interview were used to explore the characteristics and related factors of bullying behavior of teenagers on campus. Targeted preventions and interventions were put forward based on the results. In the second stage, the intervention was carried out and the effect was tested.

\subsection{Participants}

The students in a higher vocational college in Beijing participate in the research. A total of 392 valid questionnaires were collected, including 141 questionnaires for first-grade students, accounting for 36\%; 158 questionnaires for sophomores, accounting for $40.3 \%$; 93 questionnaires for third-grade students, accounting for $23.7 \%$. Among the participants, $75.4 \%$ were male and $24.6 \%$ were female. Students ranged in age from 15 to 18 . Interviewees are divided into teacher group and student group. There are 9 teacher interviewees and 8 student interviewees from

ISSN: 0010-8189

(c) CONVERTER 2020

www.converter-magazine.info 
different grades.

\subsection{Research instruments}

Instruments for interviews: The Outline of Teacher Interview includes four issues such as the bullying behavior description, characteristics analysis of bullied and be bullied, teachers' working methods and its effectiveness. The Outline of Student Interview is compiled with seven questions, involving the themes of bullying behavior description, self-experience, involvement to bullying, and suggestions.

Instruments for questionnaire: The self-designed questionnaire includes three parts, the items investigating students' demography variables and the general situation at school, "Bullying Questionnaire" and "Being Bullied Questionnaire".

Reliability test of questionnaires reveals that Crombach $\alpha$ coefficient of "Bullying Questionnaire" is 0.871 , and the Crombach $\alpha$ coefficient of "Being Bullied Questionnaire" is 0.912 . It suggests good reliability of the questionnaire. The item-total correlation coefficients are all above 0.4 , and the item-dimension correlation coefficients are all above 0.6 . It suggests good validity of the questionnaire.

\section{Results and Analysis}

3.1 Lower grades, especially freshmen, have higher degree of bullying involvement.

Chi-square test shows that the proportion of "neither bullying nor being bullied" students in three grades increases with the grade, with $72.7 \%$ in grade one, $81 \%$ in grade two and $90.3 \%$ in grade three. The distribution ratio of students involved in bully among the grades is shown in Table 1. It is consistent with the characteristics of the age difference between bullying and being bullied mentioned in the literature. The incidence of bullying and being bullied among primary and junior high school students decreases with the increase of grade, but bullying is stable in junior high school [4]. Rodlkin. (2015) indicates that bullying includes not only a well-adapted behavior, such as gaining social status by bullying, but also a dysfunctional behavior, such as immorality and behavior disorder [5]. For freshmen, adapting to the new environment is important. It may be a reason leading to higher involvement of bullying of freshmen than other grades students that freshmen try to adapt to new environment in an inappropriate manner. Therefore, improving freshmen's adaptability is important to prevent bullying behavior, and the timing of intervention should be in advance.

Table 1 Grade $\times$ Group crosstab

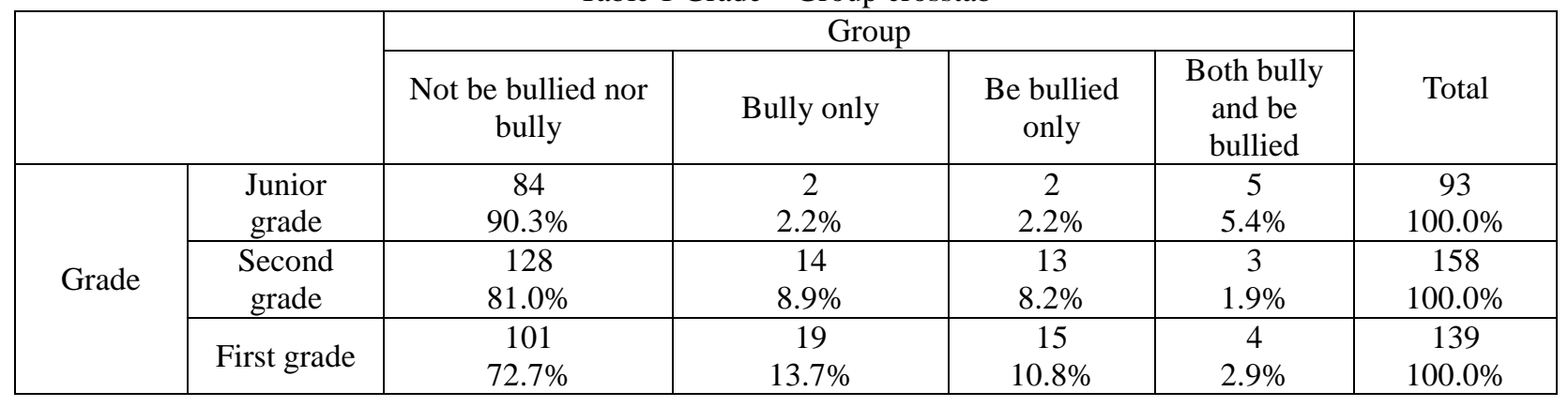

\subsection{Bullying behavior is closely related to class factors}

First, bullying behavior is mainly initiated by classmates. The survey results that the bullying students are mostly classmates, accounting for $81 \%$. Second, bullying occurs mostly in classrooms and dormitories. Bullying occurred mostly in classrooms (44.6\% reported by bullying students and $51.3 \%$ reported by bullied students), followed by dormitories (36\% reported by bullying students and $35.9 \%$ reported by bullied students). Thirdly, the mean of affection to the class is negatively correlated with the means of "Bullying Questionnaire" and "Being bullied ISSN: 0010-8189 
Questionnaire". The perceived class unity is negatively correlated with the means of "Bullying Questionnaire" and "Being bullied Questionnaire". Therefore, it is very necessary to prevent and intervene bullying from the class level.

3.3 The bullied students are most willing to ask for help from classmates or friends

When asked " Who is the person most willing to ask for help when being bullied ", $65 \%$ of the bullied students chose classmates or friends, followed by head teacher (15\%), family members (14\%), net friend (5\%)and course teachers $(1 \%)$. In the interview some teachers also mentioned that the bullied students did not actively report to the teacher, but was more willing to ask for help from the classmates around him. Therefore, it is one way of intervention to carry out bullying related education, such as class meetings, lectures, group activities. The goal is to advocacy of friendship and peace and to improve students' ability to stop bullying, including the skills of seeking help and giving help.

3.4 Interpersonal relationship is negatively correlated with frequency of bullying behavior and being bullied.

The interpersonal relationship investigated in this study includes parent-child relationship, classmate relationship and teacher-student relationship. The result shows that the mean of parent-child relationship is negatively correlated to the mean of "Bullying Questionnaire". The mean of teacher-student relationship is negatively correlated to the mean of "Bullying Questionnaire" and of "Being bullied Questionnaire". The mean of classmate relationship is negatively correlated to the mean of "Being bullied Questionnaire". It refers that students with better parent-child relationship and teacher-student relationship show less bullying behavior. Students with better classmate relationship and teacher-student relationship are less bullied. Therefore, improving students' interpersonal relationship is critical point of intervention.

3.5 The characteristics of the bullying students and being bullied students

Through interviews, the characteristics of bullying students and bullied students are summed up, so as to help us pay attention to students who may become bullying students or bullied students and intervene in advance. By improving their emotional management ability, interpersonal skills and enhancing their sense of security, perception of affiliation and self-worth, we can help them better adapt to the environment. The characteristics of bully mentioned in interview are as followed.

Teachers' view: Most of them are lack of appropriate family education: domestic violence, neglect or doting. They behave well and be sensible and enthusiastic in front of teachers. They are outgoing and righteous, enjoy power and hegemony. Some teachers think that bullying students sometimes disturb the classroom.

Students' view: They always have physical advantage: "Tall and strong", and have psychological fragility and sensitivity, poor emotional management and unconfident. Bullying students often gather to bully others and their bullying behavior has a strengthening trend.

The characteristics of the students being bullied mentioned in interview are as followed.

Teachers' view: Personality factors are greater than physiological factors, such as powerless introversion, simple and honest, quiet, no resistance to bully, lack of self-protection awareness, lack of interpersonal skills, such as "they don't know how to get along", "not talkative, not good at expressing". There are some problems in their family education such as over-protection and neglect. They distrust teachers and school, so they do not tell teachers after being bullied.

Students' view: The personality is introverted and timid, such as "retreat, afraid to talk to the bully; timid and fearful; introverted, not talkative; cowardly, incompetent". They have some disadvantages in some aspects, such as "poor learning" and "weak body". They are fragile and lack of security.

\section{Intervention Principles and Measures}

ISSN: 0010-8189

(C) CONVERTER 2020

www.converter-magazine.info 


\subsection{Principles of intervention}

Synthesize the results of questionnaire and interview, the principles of intervention are built as followed:

First, a multi-level prevention and intervention system of "school-class-individual" should be established.

As a common social problem, campus bullying must be solved not only from an individual perspective, but the most important thing is to improve or change the environment. Recent multidisciplinary research shows that the most direct and effective anti-bullying strategy should not only intervene the individual children and their family environment, but also reshape the whole campus culture and class atmosphere [6]. A study on the relationship between school moral atmosphere perception and bullying behavior shows that if students feel more caring in school, they are less likely to be involved in bullying behavior [7]. The results of this study also reflect the relationship between school system, school culture, class atmosphere, class cohesion and students' individual psychological characteristics and bullying behavior. Therefore, the intervention system should start from multiple levels and establish a multi-level prevention and intervention system of "school-class-individual".

Second, to analyze bullying behavior deeply, understand its root causes, and pay attention to both phenomena and root causes. One of the important reasons for bullying behavior is that the freshmen lack of security, trust and self-confidence. Bullying students want to get their own power by bully others, so they can protect themselves and establish their self-confidence. It is also a sign of self-protection and lack of confidence when the bullied students recoiled and did not fight back. Therefore, educators should get insight to the causes and essence behind bullying behavior. It is necessary not only to punish bullying according to the school system, but also to work on the root causes of bullying, that is, to improve the self-confidence of students, so that students can establish a sense of security and trust in the new environment sooner.

Third, focus on prevention and intervene in advance. As mentioned above, bullying can have a lot of negative effects on students' mental health and growth. In order to minimize the harm and cultivate and educate students from a positive perspective, the intervention on bullying should focus on prevention, try to do the work in advance, and reduce the incidence of bullying.

Fourth, class intervention plays an irreplaceable role. The survey results that most bullying behaviors occur within the class. Therefore, the intervention in class is the most direct and effective way to reduce bullying. Especially for freshmen, how to create a safe and friendly psychological environment for students is particularly important for classes. Class intervention can be carried out from many aspects, such as establishing and perfecting rules and regulations, enhancing class cohesion and giving full play to the role of class cadres.

Fifth, pay more attention to the mental health education for students. From the causes of bullying, students' self-worth, empathy and interpersonal skills are the key points of intervention. Existing literature shows that bullying students have low empathy ability, and it is difficult for them to feel the feelings of the bullied, which is also called "cold cognition". The bullying students get superiority and security through bullying the weak people. The results above show that bullying students generally have low self-esteem, the bullied students have a negative self-concept or self-perception. Low self-esteem and lack of confidence are reflected in their low evaluation of their intelligence and social skills, and lack of basic security in social relationships. Therefore, the intervention at the individual level can be started from three directions: improving self-worth, enhancing the empathy and improving interpersonal skills.

\subsection{Intervention program}

Based on the principles, a systematic intervention program is formulated including three levels.

\subsubsection{School level intervention}

ISSN: 0010-8189

(C) CONVERTER 2020 
(1)Psychological assessment: A general assessment of freshmen's psychological status is conducted one month after they enter school. The selected students with key concerns were interviewed. Then keeping them on concern or offering counselling or referring to other institution or hospital. Pay more attention to the students with high scores of "interpersonal sensitivity" and "hostility" as well as the students with the characteristics of the bully and the bullied.

(2)Training of Psychological Committee and Peer Counselor: When teenagers are bullied, they are most willing to ask for help from classmates or friends. Therefore, the study attempts to intervene bullying behavior by means of "peer counseling". The training of psychological committee mainly involves the identification of psychiatric mental problem, the training of communication skills, the special discussion of bullying behavior, and the guidance of class activities. In addition, peer counselors were selected and trained for 20 hours which included basic concepts and theories of group counseling, how to design group activities based on different themes for freshman classes, how to organize group activities, and some interpersonal communication strategies related to bullying behavior. The psychology committee and peer counselors are supported by supervisors during their work.

(3)Campus cultural activities: Developing campus cultural activities with mental health as the theme. Focusing on interpersonal relationships, we can improve students' mental health level and improve the cohesion of classes through various campus cultural activities. For example, some of the microfilms taken by students are about bullying. Through these activities, we can deliver positive energy and create a positive atmosphere.

(4) Strengthen the education of mental health: Popularize the knowledge of mental health, and let students learn to perceive their mental health status. Strengthen the publicity of mental health education center and psychological counseling work, help students to form a correct understanding of counseling, and guide students in need to seek help in time.

\subsubsection{Class level intervention}

(1)Head teacher training: The head teacher was trained and coached. It enhances the head teacher's understanding of bullying and improves their ability to recognize and intervene bullying. Through the training, the head teachers enhanced their ability and confidence in building the class.

(2)Curriculum: Use mental health curriculum to give specific guidance to the class. Mental health education courses are arranged after freshmen enter school. Increase the lessons of self-awareness and interpersonal relationship to quickly help freshmen adapt to college life and establish harmonious interpersonal relationships.

(3) Class group counseling: A class was selected as an experimental class, and class group counseling carried out once a week, two hours each time, and four times in total. The themes and goals of each session of group counseling are shown in Table 2.

Table 2 The themes and goals of each session of group counseling

\begin{tabular}{|l|l|l|}
\hline \multicolumn{1}{|c|}{ No. } & \multicolumn{1}{|c|}{ Themes } & \multicolumn{1}{|c|}{ Goals } \\
\hline 1 & When we are together. & Enhance class cohesion and establish group norms. \\
\hline 2 & Unity is strength. & $\begin{array}{l}\text { Improve empathy, strengthen class cohesion, and cultivate students' } \\
\text { sense of responsibility and self-confidence. }\end{array}$ \\
\hline 3 & After Rainny it is always sunny & $\begin{array}{l}\text { Improve the ability of empathy, enhance the understanding between } \\
\text { students, improve students' ability to solve interpersonal conflicts, } \\
\text { improve self-confidence. }\end{array}$ \\
\hline 4 & Cherish and love & $\begin{array}{l}\text { Enhance the emotional expression among classmates, experience } \\
\text { the love of mutual aid, and enhance the class cohesion. }\end{array}$ \\
\hline
\end{tabular}

(4)Work system of psychological committee members: psychological committee members are trained regularly, and a monthly report system of class mental health status is established. Under the guidance and supervision of full-time mental health teachers, a working system of "early detection - early intervention" is formed.

\subsubsection{Individual level intervention}

ISSN: 0010-8189

(C) CONVERTER 2020

www.converter-magazine.info 
Based on the principle of seeking help actively and voluntarily, individual psychological counseling or psychological support can be provided to the bully and the bullied under the premise of informed consent. In the actual psychological counseling work, most of the bullied students come to counseling voluntarily, and their goal in counseling is to improve their sense of self-worth, empathy and communication skills. In addition, the mental health of some bullying students who have suffered or are experiencing violence or neglect in their families is also of concern.

\section{The Effectiveness of Class Group Counseling}

Before the class group intervention, the whole class including 42 students participate the pre-test. After the group counseling, 41 students participate the post-test. Comparing the data of pre-test and post-test, the effect of class group intervention was analyzed as followed.

\subsection{The frequency of bullying and the being bullied decreased.}

There are 39 valid data in the pre-test and 41 valid data in the post-test of "Bullying Questionnaire". The results shows that after the group counseling intervention, the mean of the "Bullying Questionnaire" decreased, and the means of Physical bullying, Verbal bullying, Property bullying all decreased.

There are 42 valid data in the pre-test and 41 valid data in the post-test of "Being Bullied Questionnaire". The result shows that after intervention, the mean of "Being Bullied Questionnaire" decreased, and the means of Physical bullying, Verbal bullying, Property bullying and relational bullying all decreased.

\subsection{Students' self-worth and empathy improved.}

The result shows that the mean of students' self-worth and empathy in the post-test were higher than those in the pre-test. According to the previous literature, students' self-worth and empathy were two important factors to reduce bullying behavior. The improvement of self-worth and empathy is beneficial to reduce the occurrence of bullying.

5.3 Students' perception of affiliation of class and class unity improved.

Comparing the results of pre-test and post-test, it shows that after intervention, the experimental class students' perception of affiliation of class improved. They feel the class more united and difference between pre-test and post-test are statistically significant.

\section{Conclusion}

The influencing factors of adolescent bullying are numerous and complex, among which the grade difference of bullying reflects the adaptation needs behind the bullying, suggesting that the key time point of intervention should be placed in the enrollment stage. Many factors pointing to the class suggest that the class intervention should be regarded as a focus of bullying behavior intervention. The targeted group counseling activities for class to prevent bullying can improve students' self-worth and empathy, as well as perception of affiliation of class and the class unity, and effectively reduce the occurrence of bullying. The intervention of bullying behavior is a systematic project, which should be integrated with the functions of system guarantee, cultural edification, class management, mental health education and the multi-level intervention system of "school-class-individual" should be established.

\section{References}

[1] Z.Y. Zhu, Y. Zhou, On the interactive interpersonal relations of university students in information-based campus, Journal of China Institute of Metrology, vol. 13, no. 2, pp. 152-157, 2002.

[2] D. Olweus, "Bullying at school. Basic facts and an effective intervention programme," Promot Educ, vol. 1 , no. 4, pp. 27-31, 1994.

ISSN: 0010-8189

(C) CONVERTER 2020 
[3] W.X. Zhang, "Bullying in Primary and Middle Schools: Some Basic Facts That We Know," Journal of Shandong Normal University (Social Sciences), vol. 176, no. 3, pp. 3-8,12, 2001

[4] J.P. Liu, H. Liang, "The Present Situation and Countermeasures of Bullying Behavior about Five-year Higher Vocational College Students — Taking a Higher Vocational College in Beijing as an example," Journal of Yueyang vocational technical college, no. 6, pp. 96-99, 2015.

[5] P. C. Rodkin, D. L. Espelage, \& L. D. Hanish, "A relational framework for understanding bullying: developmental antecedents and outcomes," American Psychologist, vol. 70, no. 4, pp. 311. 2015.

[6] T.T. Chen, L.Y. Kang, "New Perspectives on School— Bullying Intervention Study," Chinese Journal of Special Education, no.7, pp. 89-92, 96, 2007.

[7] X.Y. Liang, X.T. Xue, H. Gao, D.Q. Liang, "Relationship between Perception of Morality Atmosphere in School and Behavior of Bullying among Secondary Students," China Journal of Health Psychology, vol. 20, no. 3, pp. 380-382, 2012. 